\title{
Comparison of the EFT hybrid and three-loop fixed-order calculations of the lightest MSSM Higgs boson mass
}

\author{
E. A. Reyes R.๑ and A. R. Fazio® \\ Departamento de Física, Universidad Nacional de Colombia, Ciudad Universitaria, \\ Bogotá D.C. 11001, Colombia
}

(Received 29 October 2019; published 9 December 2019)

\begin{abstract}
The lightest Higgs boson mass of the minimal supersymmetric Standard Model has been recently computed diagrammatically at the three-loop order in the whole supersymmetric parameters space of the SUSY-QCD sector. The code FeynHiggs combines one- and two-loop fixed order with the effective-fieldtheory calculations for the same Higgs mass. The two numerical predictions agree considering the scenario of only one SUSY-scale and vanishing stop mixing parameter below $10 \mathrm{TeV}$. For large SUSY scales above $10 \mathrm{TeV}$, sizeable numerical differences between the two predictions have been observed. Additionally, the combined CMS/ATLAS Higgs mass value was used to derive an upper bound on the needed SUSY scale. In the considered scenario, values above the scale $12.5 \pm 1.2 \mathrm{TeV}$ are excluded.
\end{abstract}

DOI: 10.1103/PhysRevD.100.115017

\section{INTRODUCTION}

The discovery by the ATLAS and CMS Collaborations at the CERN Large Hadron Collider (LHC) [1,2] of a bosonic particle, with properties which are compatible with those predicted for the Higgs boson of the Standard Model (SM), represents a significant progress in our understanding of the electroweak symmetry breaking mechanism. The SM is theoretically consistent with the inclusion of a $125 \mathrm{GeV}$ Higgs boson, in the sense that no Landau pole emerges, also if the model is extrapolated up to the Planck scale $\left(\Lambda_{P} \approx 10^{18} \mathrm{GeV}\right)$, where one has to accept a metastable vacuum and an unnatural high amount of fine-tuning $\left(10^{34}\right)$ for the prediction of the Higgs boson mass at the electroweak scale $\left(\Lambda_{\mathrm{EW}} \approx 10^{2} \mathrm{GeV}\right)$ [3-7]. However, there are still several puzzles that remain unsolved by the SM dynamics. The hierarchy problem, the neutrino oscillation, the identification of the dark matter, the baryon asymmetry, among others, are all left unanswered and require new physics beyond the Standard Model. The minimal supersymmetric extension of the SM (MSSM) is the best motivated and the most intensively studied framework of new physics, providing a large amount of precise predictions for experimental phenomena at the $\mathrm{TeV}$ scale $[8,9]$. In most scenarios that are phenomenologically relevant [10-13], the LHC measured value, $M_{h}^{\exp }=125.09 \pm$ $0.24 \mathrm{GeV}$ [14-16], is associated with the lightest Higgs

Published by the American Physical Society under the terms of the Creative Commons Attribution 4.0 International license. Further distribution of this work must maintain attribution to the author(s) and the published article's title, journal citation, and DOI. Funded by SCOAP. boson mass $\left(M_{h}\right)$ which is invariant under the charge conjugation and parity transformation (CP-even) and is theoretically predicted with great accuracy in the MSSM. Up to now, the dominant quantum corrections to $M_{h}$ have been computed at one-loop [17-20], two-loop [21-29], and three-loop [30-34] level using the Feynman diagrammatic (FD) and the effective potential approaches. These MSSM predictions can accommodate the measured Higgs mass value of $125 \mathrm{GeV}$ and are consistent with the similarities of the measured Higgs couplings to those in the SM [14]. Effective-field-theory (EFT) methods have been also considered to resum large logarithms in case of a large mass hierarchy between $\Lambda_{\mathrm{EW}}$ and the SUSY scale $\left(M_{\mathrm{SUSY}}\right)$ [35-41]. In particular, for values of $M_{\text {SUSY }}$ above a critical point where the fixed-order and EFT combined uncertainties are equal, the EFT computation is more accurate and therefore the usage of the SM [42] or a two-Higgsdoublet-model [43] as effective theories below the SUSY scale is preferred. Both the fixed-order and the EFT results are implemented in several publicly available codes. For the diagrammatic fixed-order calculations, there are the programs SoftSUSY [44], SUSPECT [45], CPSuperH [46], and $\mathrm{H} 3 \mathrm{~m}$ [33]. Pure EFT calculations are implemented in SUSYHD [38] and MhEFT [47]. Moreover, different hybrid methods that combine both approaches have been recently developed in order to take profit of the features of each one. FlexibleSUSY [48-50], based on SARAH [51-54], implements a hybrid method called FlexibleEFTHiggs [49,50]. This approach was also included into the program SPheno $[55,56]$. A hybrid method different from the one pursued in FlexibleEFTHiggs has been implemented in FeynHiggs $[57,58]$. There are also in literature detailed numerical comparisons between the different diagrammatic, EFT, 
and hybrid codes. In [49], it is discussed in detail how the hybrid method FlexibleEFTHiggs compares to the other EFT and diagrammatic codes. Several numerical comparisons of the hybrid approach implemented in FeynHiggs to the pure EFT calculations have been studied in $[38,49,56]$. Those papers reported surprising non-negligible numerical differences between FeynHiggs and pure EFT codes for the prediction of $M_{h}$ at large SUSY scales. The observed differences come mainly from three sources. The scheme conversion of input parameters from OS to $\overline{\mathrm{DR}}$, which can lead to large shifts due to uncontrolled higher-order terms. Unwanted effects from incomplete cancellations with subloop renormalization contributions in the determinations of the Higgs propagator pole and different parametrizations of nonlogarithmic terms. After performing the corresponding corrections, FeynHiggs results are in very good agreement with the results of SUSYHD [59]. Finally, a new hybrid computation which includes a partial $\mathrm{N}^{3} \mathrm{LL}$ resummation and $\mathrm{N}^{3} \mathrm{LO}+\mathrm{N}^{3} \mathrm{LL}$ hybrid QCD corrections to the light $C P$-even Higgs boson mass are also available [60].

For the present study, we decided to use the fixed-order and EFT hybrid calculations currently included in FeynHiggs, which seems to be in a very good agreement with the other fixed-order and EFT codes and gives a reliable three-loop predictions of the Higgs boson mass for large SUSY scales, in order to provide a numerical comparison of our three-loop fixed-order predictions of the lightest MSSM Higgs boson mass reported in [34] with the fixed-order and EFT hybrid results found in literature. As the effects of the large logarithms are expected to become relevant when $M_{\text {SUSY }}$ grows, it is natural to ask how large $M_{\text {SUSY }}$ can be. We therefore provide in this article a phenomenological analysis about the compatibility of the experimental observations at the LHC for the Higgs boson mass and the region of parameters in the specific MSSM considered scenario to find an upper bound on the needed $M_{\text {SUSY }}$.

\section{THREE-LOOP FIXED-ORDER CALCULATION OF $M_{h}$}

In contrast to the SM, the Higgs sector of the MSSM with real parameters (rMSSM) contains two complex doublets with opposite hypercharges

$$
H_{1}=\left(\begin{array}{c}
H_{1}^{0}+\frac{v_{1}}{\sqrt{2}} \\
H_{1}^{-}
\end{array}\right) \text {and } H_{2}=\left(\begin{array}{c}
H_{2}^{+} \\
H_{2}^{0}+\frac{v_{2}}{\sqrt{2}}
\end{array}\right) \text {, }
$$

where the neutral components, $H_{1,2}^{0}$ fluctuate around the vacuum expectation values (vevs) $v_{1,2}$. In the physical basis, there are five Higgs bosons, three of them are neutral: the lightest $(h)$ and heavy $(H) C P$-even Higgs bosons and the $C P$-odd Higgs boson $(A)$. The other two, $H^{ \pm}$, are charged and vev-less. Besides the SM electroweak boson masses, the rMSSM Higgs sector is parametrized in terms of two additional parameters: the mass of the $C P$-odd Higgs boson $\left(M_{A}\right)$ and $\tan \beta$, which is the ratio of the two vevs, $v_{1} / v_{2}$. The masses of the $C P$-even Higgs boson particles, $h$ and $H$, follow as predictions.

We focus in this section on the prediction of the lightest Higgs boson mass, $M_{h}$, at three-loop accuracy using a fixed-order FD computation which is based on the calculation of Higgs self-energy corrections at the given perturbative order. In this approach, the renormalized $C P$-even Higgs boson masses are obtained by finding the zeros of the determinant of the inverse propagator matrix (poles equation)

$$
\left(\Delta_{H}\right)^{-1}=-i\left(\begin{array}{cc}
p^{2}-m_{H}^{2}+\sum_{l=1}^{3} \hat{\prod}_{H H}^{(l)} & \sum_{l=1}^{3} \hat{\prod}_{h H}^{(l)} \\
\sum_{l=1}^{3} \hat{\prod}_{h H}^{(l)} & p^{2}-m_{h}^{2}+\sum_{l=1}^{3} \hat{\prod}_{h h}^{(l)}
\end{array}\right)
$$

where $m_{h}$ and $m_{H}$ denote the tree-level mass of $h$ and $H$, respectively, and

$$
\widehat{\prod}_{i j}^{(l)}=\prod_{i j}^{(l)}-\delta^{(l)} M_{i j}^{2} ; \quad i, j=h, H
$$

are the corresponding $l$-loop renormalized self-energies. A particular feature of the rMSSM is the large size of the higher-order quantum corrections to masses and couplings. They can lead to a considerably large shift on the value of the Higgs boson mass, where the bulk of the corrections comes from the SUSY-QCD sector of the Lagrangian. Thus, the dominant contributions to $\hat{\prod}_{i j}$ in Eq. (3) involve the SM parameters $h_{t}$ (top Yukawa coupling), $M_{t}$ (top quark mass), $\alpha_{s}$ (strong coupling constant), and the MSSM parameters $M_{\tilde{g}}$ (gluino mass), $\theta_{t}$ (stop mixing angle), $\tilde{m}_{q_{1,2}}$ (squark masses), and $A_{q}$ (soft breaking parameters) where $q=u, d, t, b, c, s$.

Concerning the renormalization of the self-energy corrections, that is to say, the determination of the mass counterterms $\delta^{(l)} M_{i j}^{2}$, we follow the mixed OS $/ \overline{\mathrm{DR}}$ scheme defined in [34]. Thus, the electroweak gaugeless limit at $\mathrm{O}\left(\alpha_{t} \alpha_{s}^{2}\right)$ and the approximation of zero external momentum are assumed. As a consequence, we have avoided dealing with the Higgs wave function renormalization and also with the renormalization of $\tan \beta$. Moreover, $v_{1,2}$ are defined as the minima of the full effective potential and therefore the tadpoles are renormalized on shell according to the conditions 


$$
T_{1,2}^{\text {tree }}=0, \quad \delta^{(l)} T_{1,2}=-T_{1,2}^{(l)},
$$

where $T_{j}^{(l)}$ is the $l$-loop Higgs tadpole contribution. We have also imposed an on-shell renormalization to the $A$-boson mass

$$
\delta^{(l)} M_{A A}^{2}=\operatorname{Re}\left[\prod_{A A}^{(l)}\left(M_{A}^{2}\right)\right] .
$$

The three-loop corrections of $\mathrm{O}\left(\alpha_{t} \alpha_{s}^{2}\right)$ also include the $\mathrm{O}\left(\alpha_{s}\right)$ contributions to the one-loop counterterms coming from the renormalization of the gluino mass, the top quark mass, the squark masses, and the stop mixing angles in the $\overline{\mathrm{DR}}$ scheme, as well as the two-loop $\overline{\mathrm{DR}}$ renormalization of the top mass, the stop masses, and stop mixing angles at $\mathrm{O}\left(\alpha_{s}^{2}\right)$.

For the purposes of this article, we have chosen a degenerate single-scale scenario where all the supersymmetric masses are set equal to an effective scale $M_{\text {SUSY }}$,

$$
M_{L, R}=M_{\tilde{g}}=M_{A}=\mu=M_{\mathrm{SUSY}} .
$$

Here $\mu$ is the Higgsino mass and $M_{L, R}$ are the soft SUSYbreaking masses. We have also identified the lightest Higgs boson $h$ as the SM-like Higgs boson and therefore we have assumed the decoupling limit, $M_{A}=M_{\text {SUSY }} \gg M_{t}$. This degenerate scenario in the decoupling limit is known as the "heavy SUSY" limit. As a consequence, the three-loop selfenergy corrections to $m_{h, H}^{2}$ can approximately be obtained as a superposition of the 33 vacuum integrals depicted in Fig. 1 with coefficients that are functions of the kinematic invariants and the space-time dimension, $D$. In some particular cases, the coefficients can contain poles; that is to say, terms of the form $(D-4)^{-1}$. Therefore, the basis integrals could also require a numerical evaluation of their evanescent terms. Each diagram of the basis in Fig. 1 represents a three-loop master integral of the form

$$
I_{v_{1} \ldots v_{6}}=i \frac{e^{3 \gamma_{E} \varepsilon}}{\pi^{3 D / 2}} \int \prod_{l=1}^{3} d^{D} q_{l}\left[\prod_{j=1}^{6} \frac{1}{P_{j}^{n_{j}}}\right]
$$

where

$$
\begin{aligned}
& P_{1}=q_{1}^{2}-m_{1}^{2}, \quad P_{2}=\left(q_{1}-q_{2}\right)^{2}-m_{2}^{2}, \\
& P_{3}=\left(q_{2}-q_{3}\right)^{2}-m_{3}^{2}, \quad P_{4}=q_{3}^{2}-m_{4}^{2}, \\
& P_{5}=q_{2}^{2}-m_{5}^{2}, \quad P_{6}=\left(q_{1}-q_{3}\right)^{2}-m_{6}^{2} .
\end{aligned}
$$

There are two scales involved, the electroweak scale $M_{t}$, whose associated propagator is represented with a thin solid line and the supersymmetric scale $M_{\text {SUSY }}$ represented with a thick solid line. Massless propagators are

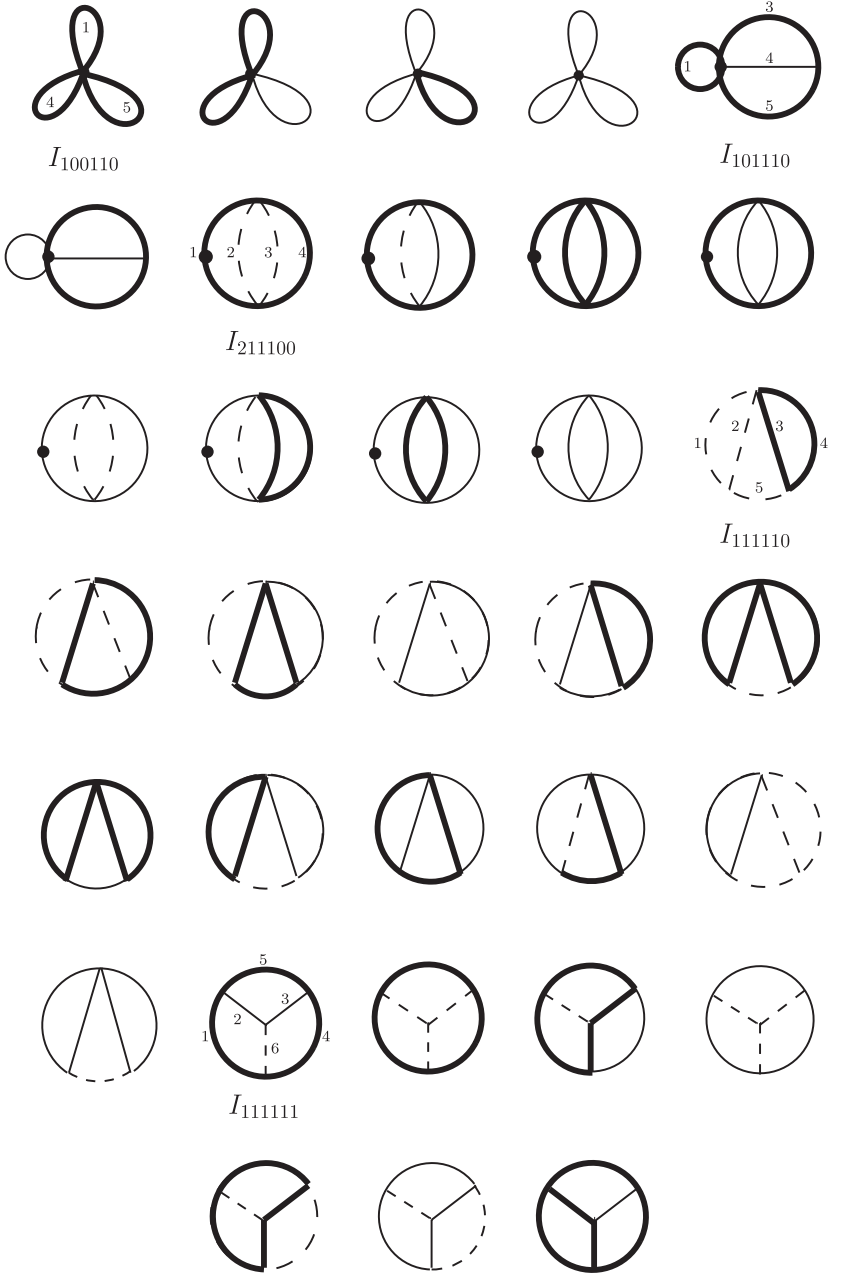

FIG. 1. Basis of three-loop master integrals. The dashed line represents a massless propagator. The thin solid line is the propagator with a mass at the electroweak scale $M_{t}$, and the thick solid line depicts the propagator involving the SUSY scale $M_{\text {SUSY }}$.

represented with a dashed line. This basis was obtained using the integration by parts (IBP) method implemented in the code Reduze [61]. Main parts of the diagram shown in Fig. 1 have been analytically evaluated in [62-69]. The numerical evaluation of the basis integrals was done with the programs TVID [70,71] and SecDec [72]. In particular, the integral $I_{211100}$ requires a Laurent expansion up to first order in $\varepsilon$. The evanescent terms of $\mathrm{O}\left(\varepsilon^{1}\right)$ was numerically evaluated with the help of SecDec.

\section{EFT HYBRID CALCULATION OF $\mathrm{M}_{h}$}

When there is a large mass hierarchy between the electroweak scale and the scale of the SUSY particles, the fixed-order computations of the Higgs self-energy corrections contain large logarithms that can spoil the convergence of the perturbative expansion and yield unreliable predictions of the Higgs boson masses. A fixed-order computation 
is thus recommended for low values of $M_{\mathrm{SUSY}}$ not separated too much from $M_{t}$. There is an alternative approach to calculate $M_{h}$ which yields accurate results for high SUSY scales. This approach is based on the EFT techniques [38,73] and allows the resummation of the large logarithmic terms and the incorporation of higher-order contributions beyond the order of the fixed-order diagrammatic calculations. In the heavy SUSY limit, the low-scale EFT below $M_{\text {SUSY }}$ is the SM. It requires just one EFT coupling, the effective Higgs self coupling $\lambda$, which correlates the high scale $M_{\text {SUSY }}$ and the low scale $M_{t}$ through the renormalization group equations (RGEs) and captures radiative corrections of the form

$$
\alpha_{j}^{n+m-1} \log ^{n}\left(M_{\mathrm{SUSY}} / M_{t}\right) ; \quad j=\lambda, h_{t}, g_{s}, \ldots
$$

for $n \geq 1$, by using the $m$-loop beta functions of $\alpha_{j}$, into the running coupling $\lambda(Q)$. In order to get a SM running Higgs mass in the $\overline{\mathrm{MS}}$ scheme at the scale $M_{t}$, one has to multiply $\lambda\left(M_{t}\right)$ by $2 v^{2}\left(M_{t}\right)$, where $v\left(M_{t}\right) \approx 246 \mathrm{GeV}$ is the $\overline{\mathrm{MS}} \mathrm{vev}$ evaluated at $M_{t}$. The physical Higgs mass requires to solve the pole equation

$$
p^{2}-2 \lambda\left(M_{t}\right) v^{2}\left(M_{t}\right)+\widetilde{\prod}_{h h}^{\mathrm{SM}}\left(p^{2}\right)=0,
$$

with the SM Higgs boson self-energy,

$$
\widetilde{\prod}_{h h}^{\mathrm{SM}}\left(p^{2}\right)=\left[\prod_{h h}^{\mathrm{SM}}\left(p^{2}\right)-\frac{1}{\sqrt{2} v} T_{h}^{\mathrm{SM}}\right]_{f i n}
$$

renormalized in the $\overline{\mathrm{MS}}$ scheme but with the Higgs tadpoles renormalized to zero, i.e., $\delta T_{h}^{\mathrm{SM}}=-T_{h}^{\mathrm{SM}}$. As higher dimensional operators are not included into the effective Lagrangian, the contributions suppressed by the heavy scale $M_{\text {SUSY }}$ are not considered. Consequently, the EFT calculation is less accurate than the fixed-order one for low SUSY scales. The fixed-order calculation is more accurate below a critical SUSY mass scale, estimated to be about $M_{\text {SUSY }}^{C} \approx$ 1.2 TeV in [42], whereas above that scale the EFT calculation is more accurate.

In the version 2.14.3 of FeynHiggs [74], both approaches are combined in order to supplement the full one-loop, leading, and subleading two-loop diagrammatic results with a resummation of the leading + next to leading (LL + NLL) [75] and next to next to leading (NNLL) [76] logarithmic contributions coming from the top/stop sector. For the resummation of large logarithms up to NLL two-loop RGEs and one-loop matching conditions are needed, accordingly, the resummation up to NNLL requires three-loop RGEs and two-loop matching conditions. The hybrid results obtained from the combination of the two approaches are added into the pole equation of the full MSSM

$$
p^{2}-m_{h}^{2}+\tilde{\prod}_{h h}\left(p^{2}\right)+\Delta_{h h}^{\log }=0
$$

through the shift $\Delta_{h h}^{\log }$ which contains the resummed large logarithms from the EFT as well as the logarithmic terms already present in the fixed-order Higgs self-energies

$$
\Delta_{h h}^{\log }=-\left[2 \lambda\left(M_{t}\right) v^{2}\left(M_{t}\right)\right]_{\log }-\left[\tilde{\prod}_{h h}\left(m_{h}^{2}\right)\right]_{\log } .
$$

The subscript $\log$ means that only logarithmic terms are considered. The logarithms in the Higgs self-energy appear explicitly only after expanding in $v / M_{\mathrm{SUSY}}$. This subtraction term ensures that the one- and two-loop logarithms, already contained in the fixed-order FD computation, are not counted twice. In general, the higher-order logarithms obtained from the EFT and the hybrid approaches are not the same because the determination of the poles of the propagators [Eqs. (9) and (11)] are performed in different models. However, this difference, which comes from the momentum dependence of the two-loop order non-SM contributions to the Higgs self-energy, cancels out with contributions coming from the subloop renormalization in the heavy SUSY limit, as was explicitly shown in [59]. Besides the unwanted effects from incomplete cancellations in the determination of the Higgs propagator pole, the effects due to nonlogarithmic terms and its parametrization as well as the higher-order terms coming from the scheme conversion between $O S$ and $\overline{\mathrm{DR}}$ parameters are all included into FeynHiggs 2.14 [74].

\section{NUMERICAL RESULTS}

In this section, we present a numerical comparison of our three-loop fixed-order predictions of $M_{h}$ to the numerical predictions coming from the version 2.14.3 of FeynHiggs. We have chosen a $\overline{\mathrm{DR}}$ renormalization of the stop sector with the renormalization scale set to $\mu_{r}=M_{\mathrm{SUSY}}$, which is equivalent to set $Q_{t}=-1$ in FeynHiggs. In the $\overline{\mathrm{DR}}$ scheme, the two-loop anomalous dimension of the stop mass contains a nonphysical dependence on the $\varepsilon$-scalar mass, $m_{\varepsilon}$. At order $\alpha_{t} \alpha_{s}^{2}$, a one-loop renormalization of $m_{\varepsilon}$ is required. In order to decouple the $\varepsilon$-scalar mass from the physical observables, we have renormalized $m_{\varepsilon}$ in the onshell scheme [77] and we have imposed $m_{\varepsilon}^{O S} \rightarrow 0$ at the given perturbative order. Strictly speaking, this procedure does not coincide with a $\overline{\mathrm{DR}}$ renormalization due to $m_{\varepsilon}$ is not renormalized minimally. However, as all the other parameters entering the two-loop counterterm of the stop mass are $\overline{\mathrm{DR}}$ parameters and the $\mathrm{O}\left(\alpha_{t} \alpha_{s}^{2}\right)$ correction to $M_{h}$ is independent from $m_{\varepsilon}$, we maintain the nomenclature

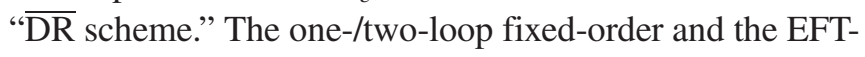
hybrid FeynHiggs predictions are fixed such that the full MSSM is considered (mssmpart $=4$ ) in its real version (higgsmix $=2$, tlCplxApprox $=0$ ), no approximation is 
taken for the one-loop result $(\mathrm{p} 2$ approx $=4)$, and the $O\left(\tan ^{n} \beta\right)$ corrections are resummed (botResum $=1$ ). In particular, when the resummation of the large logarithms is included, we use the full LL, NLL, and NNLL resummation (looplevel $=2$, loglevel $=3$ ). The top quark mass is renormalized in the SM $\overline{\mathrm{MS}}$ scheme at NNLO (runningMT $=1$ ) since for loglevel different from zero a $\overline{\mathrm{DR}}$ renormalization is not allowed. To obtain the pole mass $M_{h}$ at three-loop level in the fixed-order approach, we have introduced the $O\left(\alpha_{t} \alpha_{s}^{2}\right)$ corrections as constant shifts in the FeynHiggs 1-loop +2 -loop Higgs renormalized selfenergies (looplevel $=2$ and loglevel $=0$ ) with the help of the function FHAddSelf, but in this case we have used a $\overline{\mathrm{DR}}$ renormalization of the top quark mass. In order to assure a correct evaluation of the parameters $\alpha_{s}$ and $M_{t}$ in the $\overline{\mathrm{DR}}$ scheme at the desired perturbative order, we have used the code decSUSY which is a supersymmetric extension of the program RunDec [78-80]. We start by considering the FeynHiggs fixed-order, FeynHiggs NNLL hybrid and three-loop $O\left(\alpha_{t} \alpha_{s}^{2}\right)$ predictions. The upper plot of Fig. 2 shows the dependence of $M_{h}$ on $M_{\text {SUSY }}$ for a vanishing stop mixing, $X_{t} / M_{\mathrm{SUSY}}=0$, at the kinematic point $A_{e, \mu, \tau, u, d, c, s, b}=0$ and $\tan \beta=10$, whereas the lower plot shows the numerical differences between all the considered FeynHiggs results and the $O\left(\alpha_{t} \alpha_{s}^{2}\right)$ prediction of $M_{h}$. In order to draw these plots, we have adopted the heavy SUSY limit [Eq. (6)] and we have followed the next conventions. The one- and two-loop fixedorder results of FeynHiggs are represented with the dotdashed and the dashed lines, respectively. The blue dotted line contains, in addition, the resummation of the large logarithms up to NNLL order. The blue band corresponds to the uncertainty in the NNLL prediction computed with the help of the FeynHiggs function FHUncertainties for the flag choice: mssmpart $=4$, looplevel $=2$, loglevel $=3$, runningMT $=1$. In principle, three effects are taken into account: (i) the variation of the renormalization scale from $M_{t} / 2$ to $2 M_{t}$, (ii) the use of $M_{t}^{\text {pole }}$ instead of $M_{t}^{\text {run }}$ in the two-loop corrections, and (iii) the exclusion of higher-order resummation effects in $M_{b}$. The brown band is the experimental Higgs boson mass and its corresponding uncertainty; we have adopted the combined CMS/ ATLAS result of the RUN 1 at the LHC, $M_{h}^{\text {exp }}=125.09 \pm$ $0.24 \mathrm{GeV}$ [14], since there is not yet an official combined result for RUN $2[15,16]$ observations. Finally, the red solid line contains our three-loop fixed-order corrections.

The first thing to note here (and also in Fig. 3) is that the higher-order large logarithms coming from the EFT hybrid approach at NNLL level produce a growing positive shift on the two-loop predictions reaching a size of about $20 \mathrm{GeV}$ for $M_{\text {SUSY }}=40 \mathrm{TeV}$. Additionally, the NNLL predictions are in a very good agreement with the threeloop $O\left(\alpha_{t} \alpha_{s}^{2}\right)$ results for $M_{\text {SUSY }}$ less than the value $M_{\text {SUSY }} \lesssim 10 \mathrm{TeV}$. On the lower graph of Fig. 2, one can
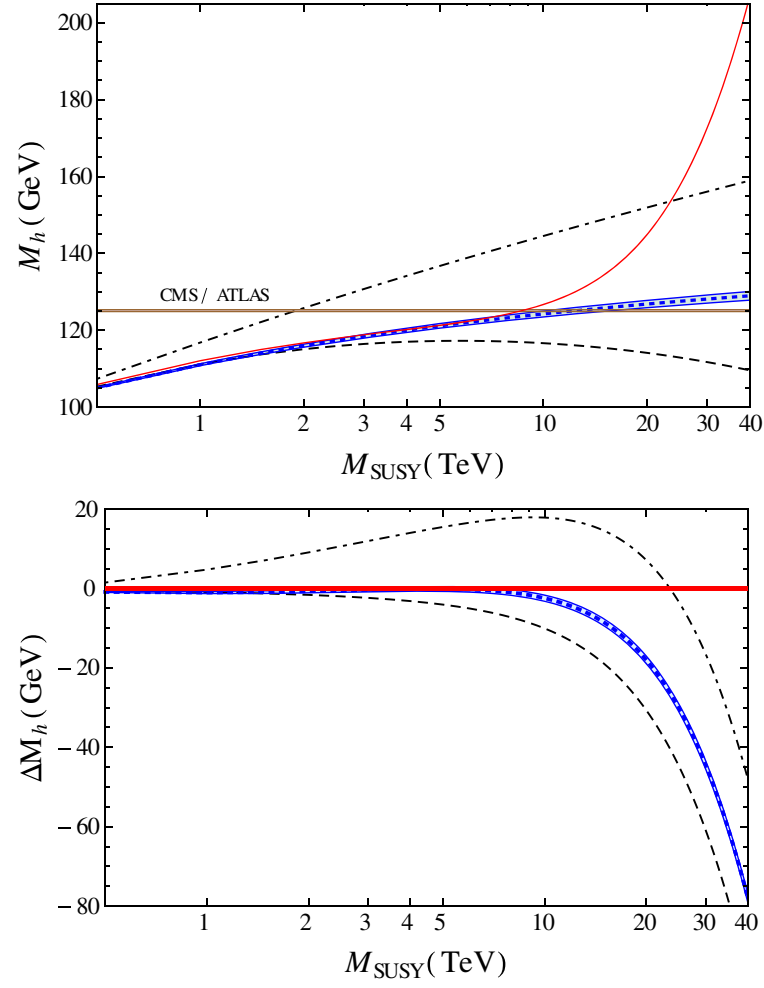

FIG. 2. Comparison of the $M_{h}$ predictions of FeynHiggs with the three-loop fixed-order computation of $M_{h}$ at $O\left(\alpha_{t} \alpha_{s}^{2}\right)$ in the heavy SUSY limit. The dot-dashed and the dashed lines are the fixed-order results of FeynHiggs at one and two-loop level, respectively. The blue dotted line contains the NNLL resummation of the large logarithms in FeynHiggs. The blue band corresponds to the uncertainty in the NNLL prediction taken from FeynHiggs. The brown band is the CMS/ATLAS Higgs boson mass, $M_{h}^{\exp }=125.09 \pm 0.24 \mathrm{GeV}$. The red solid line represents our three-loop fixed-order predictions. Up: dependence of $M_{h}$ on the supersymmetric scale $M_{\text {SUSY }}$ for a vanishing stop mixing, $X_{t} / M_{\text {SUSY }}=0$. Down: numerical differences between the FeynHiggs predictions and the three-loop fixed-order predictions of $M_{h}$.

see that in the region $2.2 \mathrm{TeV} \lesssim M_{\text {SUSY }} \lesssim 7.4 \mathrm{TeV}$ there is an approximately constant difference of about $0.2 \mathrm{GeV}$ between the red solid and the blue dotted line which is within the theoretical uncertainty (blue band) estimated to be about $0.6 \mathrm{GeV}$. Below this region the agreement is still good with a numerical difference of at most $1 \mathrm{GeV}$. However, for scales above $10 \mathrm{TeV}$, the effects of the large logarithms in the red curve start to be relevant; the difference between the two results rapidly increases up to $\sim 21 \mathrm{GeV}$ when $M_{\text {SusY }}$ grows to up to $20 \mathrm{TeV}$ and grows monotonically reaching $78 \mathrm{GeV}$ at $M_{\text {SUSY }}=40 \mathrm{TeV}$. This pronounced behavior depends crucially on our election of the input parameters $\mu_{r}, M_{\tilde{g}}$, and $X_{t}$. The presence of $n$-loop logarithms of the form $\log ^{n}\left(M_{\mathrm{SUSY}} / M_{t}\right)$ in the master integrals of Fig. 1 can introduce additional large contributions in the three-loop predictions of $M_{h}$. 

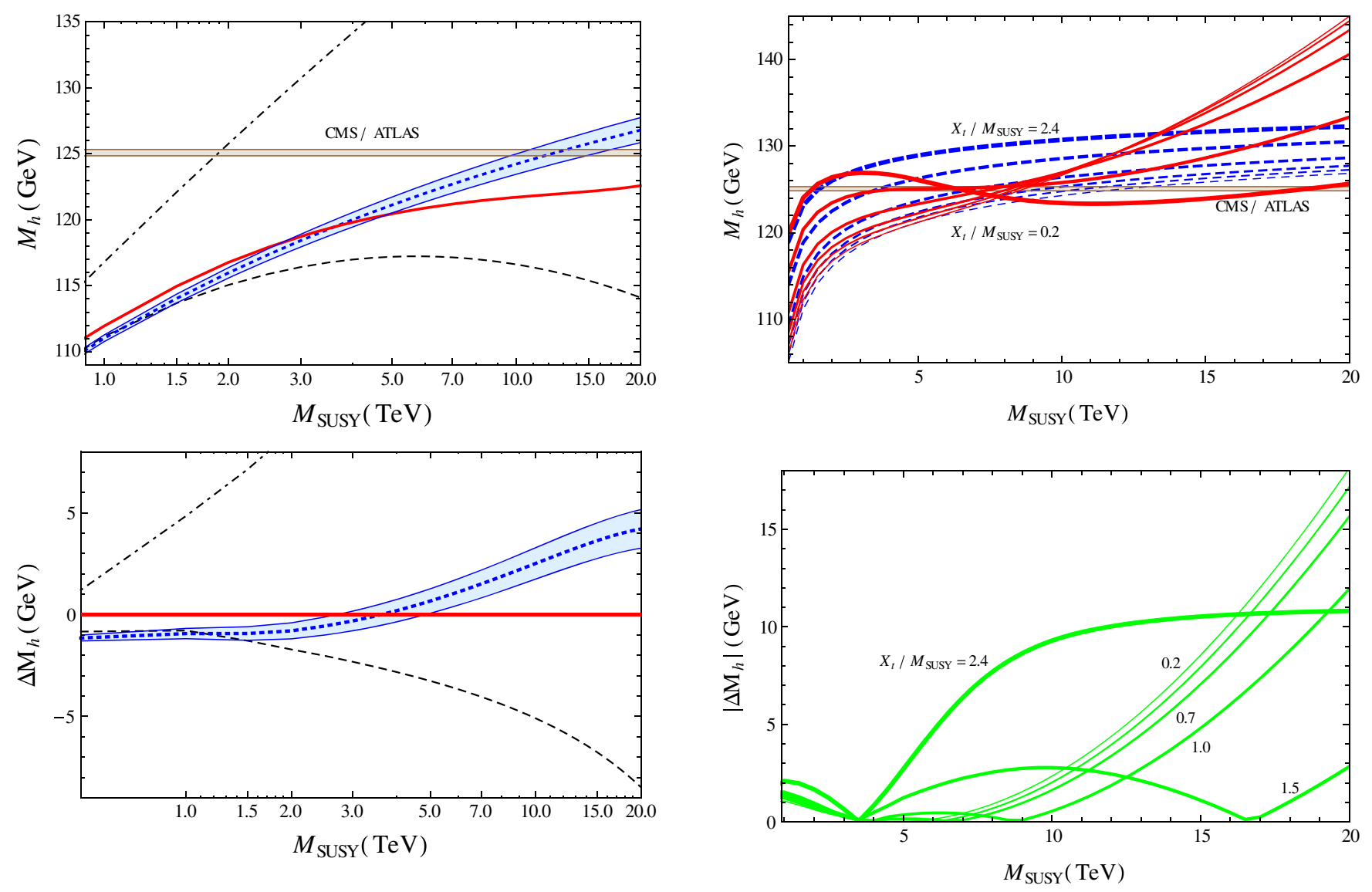

FIG. 3. Numerical comparison of the $M_{h}$ predictions in a scenario where $M_{\tilde{g}}=1.5 \mathrm{TeV}$ and $X_{t} / M_{\text {SUSY }}=0$. These plots follow the same conventions as in the Fig. 2. Up: evolution of $M_{h}$ as a function of $M_{\text {SUSY }}$. Down: differences between the threeloop fixed-order and the FeynHiggs predictions.

In Fig. 3, we show the numerical comparison between two results. (i) Our three-loop $\mathrm{O}\left(\alpha_{t} \alpha_{s}^{2}\right)$ predictions of $M_{h}$ (red curve), where the heavy SUSY limit has been smoothed to include an additional SUSY scale, the gluino mass $M_{\tilde{g}}$. (ii) The FeynHiggs prediction of $M_{h}$ including the resummation of the large logarithms up to NNLL order (blue dashed curve). The effects of a gluino threshold are not included in the NNLL resummation procedure since three-loop RGEs for an appropriate extension of the Standard Model with the gluino as additional fermion, for instance, as a singlet of the gauge group, are not included in FeynHiggs. The NNLL resummation is thus restricted to the case $M_{\tilde{g}}=M_{\mathrm{SUSY}}$. However, the numerical effects due to a gluino threshold in the EFT computation of $M_{h}$ are numerically small, about $0.2 \mathrm{GeV}$ [76], and therefore can be safely neglected. The fixed-order corrections instead capture almost the entire effect of varying $M_{\tilde{g}}$. In [76] was shown that the diagrammatic two-loop correction to $M_{h}$ gives a sizable contribution of up to $\sim 2 \mathrm{GeV}$ for the case of maximal stop mixing. The three-loop fixed-order corrections with the added gluino can also be sizeable, specially for large SUSY scales. We have considered a

FIG. 4. Numerical comparison of the $M_{h}$ predictions for a nonvanishing stop mixing in the heavy SUSY limit. The blue dashed lines are the NNLL predictions of FeynHiggs, and the red solid lines represent our three-loop fixed-order predictions. The brown band is the CMS/ATLAS Higgs boson mass, $M_{h}^{\exp }=$ $125.09 \pm 0.24 \mathrm{GeV}$. Up: $M_{h}$ as a function of $M_{\mathrm{SUSY}}$ for different stop mixing values, $X_{t} / M_{\text {SUSY }}=0.2,0.5,0.7,1.0,1.5$, and 2.4. Down: absolute numerical differences between the three-loop fixed-order predictions and the NNLO results of FeynHiggs plotted in the upper figure.

gluino mass of $M_{\tilde{g}}=1.5 \mathrm{TeV}$. The inclusion of this additional scale produces significant differences between the $O\left(\alpha_{t} \alpha_{s}^{2}\right)$ and the NNLL results. Note that the red curve includes not only the gluino effects but the complete dynamics, that is to say, the large logarithms of the form $\ln \left(M_{\mathrm{SUSY}} / M_{t}\right)$ and $\ln \left(M_{\mathrm{SUSY}} / M_{\tilde{g}}\right)$ are included. For small SUSY scales below $\sim 3.5 \mathrm{TeV}$, the difference is always less than $1.3 \mathrm{GeV}$. For large SUSY scales $\left(M_{\mathrm{SUSY}}>3.5 \mathrm{TeV}\right)$, this difference grows to a maximum value of $4 \mathrm{GeV}$ when $M_{\text {SUSY }}=20 \mathrm{TeV}$. Nevertheless, the numerical effect of the large logarithms in the red curve is reduced by a factor of around 5 regarding the results shown in Fig. 2.

Finally, we have studied the dependence of the NNLL and three-loop $M_{h}$ predictions on the stop mixing parameter $X_{t}$ in the heavy SUSY limit.

In Fig. 4, we have increased the value of $X_{t} / M_{\text {SUSY }}$ from 0.2 (thin curves) to 2.4 (thick curves). We observe a good 
agreement between the two predictions for small SUSY scales up to $M_{\text {SUSY }}=10 \mathrm{TeV}$, which is in accordance with previous comparisons of fixed-order and EFT calculations $[40,60]$. However, for high energy scales above $M_{\text {SUSY }} \gtrsim 10 \mathrm{TeV}$, there is a large variation of the threeloop fixed-order curves which is observed neither in the NNLL FeynHiggs curves nor in the three-loop EFT and hybrid results presented in $[33,40,60]$ where the resummation of the large logarithmic contributions is included. In particular, when $X_{t} / M_{\text {SUSY }}$ is equal to 1.5 , which is an inflexion point where the curvature of $M_{h}\left(X_{t}\right)$ changes its sign, the numerical differences between the red and the blue curves remain lesser than $3 \mathrm{GeV}$ up to $M_{\text {SUSY }}=20 \mathrm{TeV}$. In the case of maximal stop mixing, $X_{t} / M_{\mathrm{SUSY}}=2.4$, where the prediction of $M_{h}$ takes its higher value (thickest lines in Fig. 4), the difference between the two results is almost constant for large SUSY scales amounting a size of about $10 \mathrm{GeV}$. The other considered $X_{t}$ values show a numerical difference that grows without boundary as a function of $M_{\mathrm{SUSY}}$. Due to the large variation in the red and green curves, it is not possible to observe a systematic improvement of the effect of the large logarithms in the three-loop fixed-order results when $X_{t}$ is changed.

We further explore the dependence of the Higgs boson mass on the SUSY input parameters $M_{\mathrm{SUSY}}, X_{t}$, and $\tan \beta$ in the heavy SUSY limit. Figures $2-4$ show that the predicted value of $M_{h}$ grows when $M_{\text {SUSY }}$ increases and reaches a maximum value at the critical point $X_{t} / M_{\text {SUSY }}=2.4$, whose location is independent of $M_{\text {SUSY }}$. It suggests that one can find boundaries for the region of rMSSM parameters which put further constraints on $M_{h}$. Figure 5 shows the numerical values of $X_{t} / M_{\mathrm{SUSY}}$ and $M_{\mathrm{SUSY}}$ which produce the same Higgs mass prediction (gray curves). We have considered values of $M_{h}$ from 115 to $131 \mathrm{GeV}$ and set $\tan \beta=10$. We observed here that there is a minimum value of $M_{\mathrm{SUSY}}$, located at the maximal point $X_{t} / M_{\mathrm{SUSY}}=$ 2.4, which is compatible with some election of the Higgs boson mass. Moreover, in the case of nonstop mixing $\left(X_{t}=0\right)$, one can find the higher value of $M_{\text {SUSY }}$ compatible with a given $M_{h}$. These extrema values grow when we consider higher values of $M_{h}$. This behavior can also be seen at the intersection of the brown band with the blue dashed lines in Fig. 4 for a $125 \mathrm{GeV}$ Higgs mass. If we use the combined CMS/ATLAS measured Higgs boson mass within the actual combined uncertainties, $M_{h}^{\exp }=125.09 \pm$ $0.24 \mathrm{GeV}$, we will be able to fix upper and lower bounds on the SUSY scale $M_{\text {SUSY }}$ in the benchmark scenario considered in this work.

Figure 6 shows the $125.09 \mathrm{GeV}$ contours (gray lines) as a function of $M_{\mathrm{SUSY}}, \tan \beta$ (up: for values of $X_{t} / M_{\mathrm{SUSY}}$ from 0 to 2.4) and $X_{t} / M_{\text {SUSY }}$ (down: for values of $\tan \beta$ from 4 to 30 ). The blue and the brown regions refer to the SUSY parameters compatible with $M_{h}^{\exp }$. The purple lines represent the combined uncertainty for the cases enclosed inside. Notice that if $\tan \beta \leq 10$ then $M_{\text {SUSY }}$ strongly

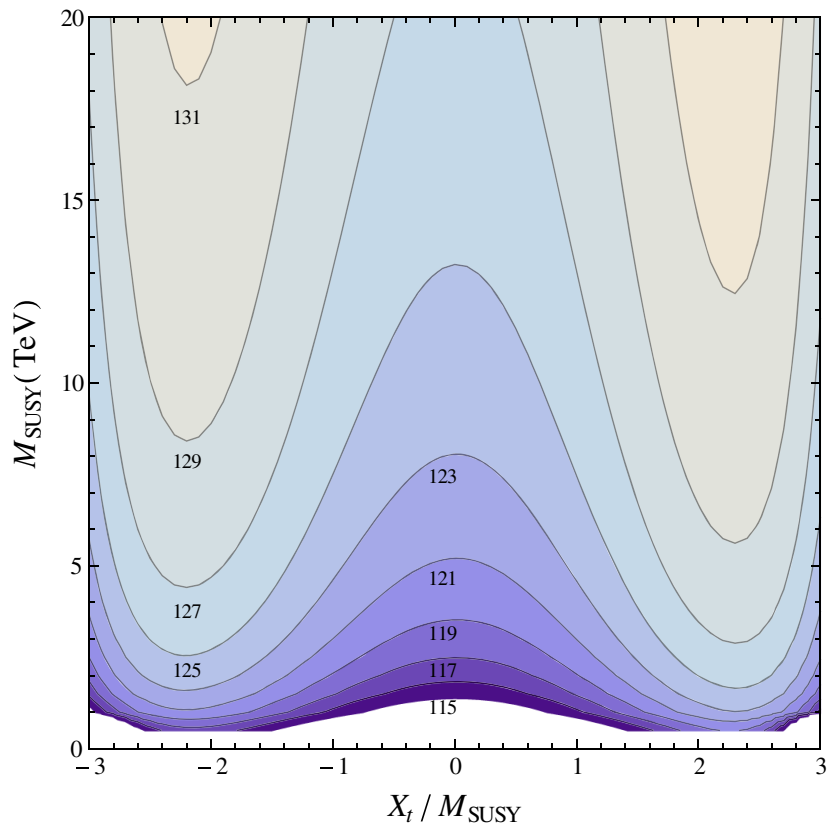

FIG. 5. Dependence of $M_{h}$ on $M_{\text {SUSY }}$ and $X_{t}$ in the heavy SUSY limit. We have used $\tan \beta=10$. The gray lines represent the values of $M_{\text {SUSY }}$ and $X_{t}$ which produce the same Higgs boson mass. The predicted value of $M_{h}$ increases monotonically with $M_{\text {SUSY }}$.

depends on $\tan \beta$; moreover, the parameter region of $\tan \beta \lesssim 3$ is incompatible with the LHC observations of the Higgs boson mass if one considers SUSY scales below $20 \mathrm{TeV}$. For values above 10, the dependence is marginal and the curves flatten. In general, it is not possible to find a global upper bound on the required SUSY scale, from the CMS/ATLAS Higgs mass value, which is independent of the election of the parameters $X_{t}$ and $\tan \beta$. However, for large $\tan \beta$ values ( $\tan \beta \gtrsim 10$ ), owing to the curves are almost constant, one can identify a lower bound for $X_{t} / M_{\text {SUSY }}=2.4$ and an upper bound for a vanishing stop mixing parameter $\left(X_{t}=0\right)$. When $\tan \beta=10$, which is the point considered in all the above plots of this section, we find that $M_{\text {SUSY }}$ must be at most $12.5 \pm$ 1.2 $\mathrm{TeV}$ (see purple line in the upper plot) in order to be in agreement with the CMS/ATLAS experimental results. $M_{\text {SUSY }}$ can be reduced up to $9.6 \mathrm{TeV}$ for $\tan \beta=30$ and $X_{t}=0$. One can significantly lower the required value of $M_{\text {SUSY }}$ to $1.2 \mathrm{TeV}$ when $\left|X_{t} / M_{\text {SUSY }}\right|$ increases up to 2.4 and for $\tan \beta=30$.

There is still the possibility to use the vacuum stability of the Higgs potential to find a global upper bound on $M_{\text {SUSY }}$ including small $\tan \beta$ values, as was discussed in [42]. The estimated bound obtained in this work is of the order of $3.7 \times 10^{11} \mathrm{GeV}$; however, this result is not conclusive because it was derived only for $\tan \beta=1$ without including the full MSSM Higgs potential and is valid in the $\overline{\mathrm{DR}}^{\prime}$ scheme, where $m_{\varepsilon}$ is minimally renormalized and its dependence is decoupled from the observables through appropriated shifts of the physical parameters [81,82]. 

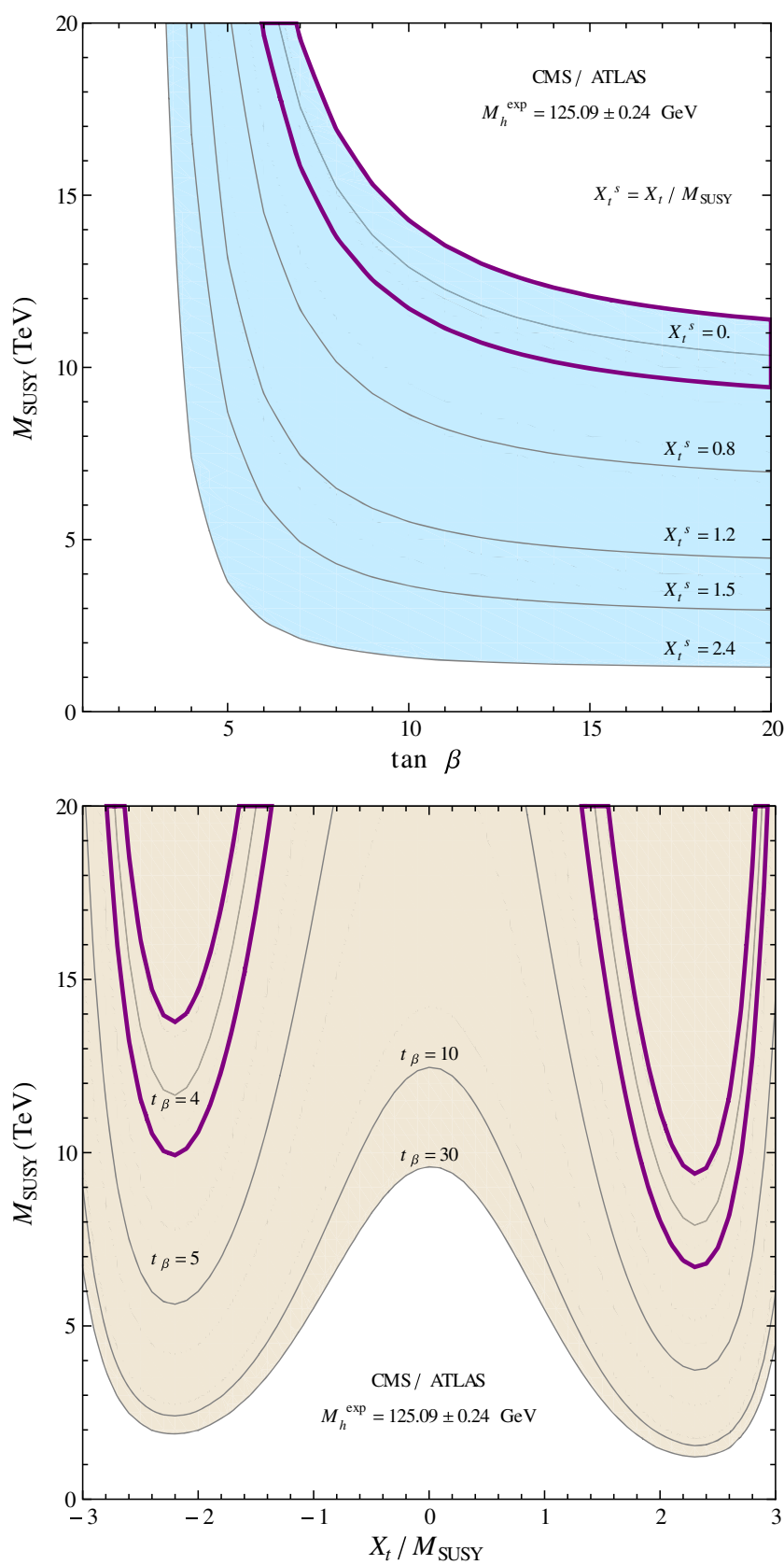

FIG. 6. Region of rMSSM parameters in the heavy SUSY limit which is compatible with the central value and the combined uncertainty of the CMS/ATLAS Higgs boson mass, $M_{h}^{\exp }=$ $125.09 \pm 0.24 \mathrm{GeV}$. Up: gray lines represent the points $\left(M_{\mathrm{SUSY}}\right.$, $\tan \beta$ ) compatible with a $125.09 \mathrm{GeV}$ Higgs mass for different values of the stop mixing parameter, $X_{t} / M_{\mathrm{SUSY}}=0,0.8,1.2,1.5$, 2.4. The purple line represents the combined uncertainty for the case of zero stop mixing. Down: gray lines are the $125.09 \mathrm{GeV}$ contours as a function of $M_{\mathrm{SUSY}}$ and $X_{t} / M_{\mathrm{SUSY}}$ for different values of the parameter $\tan \beta, \tan \beta=4,5,10,30$. The purple lines are the points compatible with the combined uncertainty for the lowest value of $\tan \beta$ considered.

A complete phenomenological analysis in the $\overline{\mathrm{DR}}$ scheme including small $\tan \beta$ values and the full MSSM Higgs potential is missing and is beyond the scope of this paper.
The region $M_{\text {SUSY }}>12.5 \pm 1.2 \mathrm{TeV}$, where the threeloop fixed-order results blow up, is excluded by the combined CMS/ATLAS Higgs mass value in the simple scenario consider here. The coming combined result for RUN 2 by ATLAS and CMS will reduce the current uncertainty, and therefore the upper bound on the SUSY scale (for higher values of $\tan \beta$ ) could be reduced even more.

\section{CONCLUSIONS}

We have recently presented a fixed-order computation of the lightest rMSSM Higgs boson mass which extends the validity of the leading three-loop corrections to the whole parameter space of the rMSSM [34]. This computation is in a very good agreement with the results of $\mathrm{H} 3 \mathrm{~m}$ [33] for low SUSY scales $\left(M_{\text {SUSY }} \lesssim 1.2 \mathrm{TeV}\right)$. However, for large $M_{\text {SUSY }}$, a numerical comparison with the available codes is missing. We have decided to fill this gap by checking our computation of $M_{h}$ with the three-loop results coming from the EFT hybrid approach implemented in FeynHiggs 2.14 [74] for the same observable. FeynHiggs includes the resummation of the large logarithms at high SUSY scales and is in a very good agreement with the other fixedorder and EFT codes. This allowed us to compare our results with a reliable three-loop $M_{h}$ prediction for $M_{\mathrm{SUSY}}$ up to $20 \mathrm{TeV}$. We focused on a single SUSY scale scenario in the decoupling limit (heavy SUSY limit) where the SM is the low energy EFT. We specifically compared our $O\left(\alpha_{t} \alpha_{s}^{2}\right)$ and the FeynHiggs NNLL predictions of $M_{h}$ at the kinematical point $A_{e, \mu, \tau, u, d, c, s, b}=0, \tan \beta=10$ and $\mu_{r}=M_{\text {SUSY }}$. We find a very good agreement between the two results for SUSY scales below $10 \mathrm{TeV}$ in the case of vanishing stop mixing $\left(X_{t}=0\right)$. The difference is estimated to be in the range $0.2 \mathrm{GeV} \lesssim \Delta M_{h} \lesssim 1 \mathrm{GeV}$ for the region $M_{\text {SUSY }} \lesssim 10 \mathrm{TeV}$. Above $M_{\text {SUSY }}=10 \mathrm{TeV}$ we have observed significant differences that increase monotonically with $M_{\text {SUSY }}$. Such a behavior is expected for high SUSY scales since the $O\left(\alpha_{t} \alpha_{s}^{2}\right)$ computation contain the effects of the large logarithmic contributions. The numerical differences can be reduced through the introduction of an additional SUSY scale, the gluino mass $M_{\tilde{g}}$. The variation of the stop mixing parameter $X_{t}$ does not produce a systematic improvement of the large logarithm effects in the three-loop fixed-order corrections. Nevertheless, the region where the contributions of the large logarithms blow up is excluded by the combined CMS/ATLAS Higgs mass, $M_{h}^{\exp }=125.09 \pm 0.24 \mathrm{GeV}$. This exclusion is valid just for large $\tan \beta$ values, $\tan \beta \gtrsim 10$, where we have derived an upper bound on the needed SUSY scale. For values above $\tan \beta=10$, the region $M_{\mathrm{SUSY}}>12.5 \pm 1.2 \mathrm{TeV}$ is ruled out. For small $\tan \beta$ values, upper bounds of $M_{\mathrm{SUSY}}$ could not be found in the SUSY parameter region considered in this work. 


\section{ACKNOWLEDGMENTS}

This work is partially financially supported by the research Grant No. 39844 of the call CONVOCATORIA 727 DE COLCIENCIAS PARA DOCTORADOS NACIONALES 2015.

[1] G. Aad et al. (ATLAS Collaboration), Phys. Lett. B 716, 1 (2012).

[2] S. Chatrchyan et al. (CMS Collaboration), Phys. Lett. B 716, 30 (2012).

[3] G. Degrassi, S. Di Vita, J. Elias-Miró, J. R. Espinosa, G. F. Giudice, G. Isidori, and A. Strumia, J. High Energy Phys. 08 (2012) 098.

[4] D. Buttazzo, G. Degrassi, P. P. Giardino, G. Giudice, F. Sala, A. Salvio, and A. Strumia, J. High Energy Phys. 12 (2013) 089.

[5] F. Bezrukov, M. Yu. Kalmykov, B. A. Kniehl, and M. Shaposhnikov, J. High Energy Phys. 10 (2012) 140.

[6] B. A. Kniehl, A. F. Pikelner, and O. L. Veretin, Nucl. Phys. B896, 19 (2015).

[7] A. V. Bednyakov, B. A. Kniehl, A. F. Pikelner, and O. L. Veretin, Phys. Rev. Lett. 115, 201802 (2015).

[8] H. P. Nilles, Phys. Rep. 110, 1 (1984).

[9] H. E. Haber and G. L. Kane, Phys. Rep. 117, 75 (1985).

[10] S. Heinemeyer, O. Stål, and G. Weiglein, Phys. Lett. B 710, 201 (2012).

[11] M. Carena, S. Heinemeyer, O. Stål, C. E. M. Wagner, and G. Weiglein, Eur. Phys. J. C 73, 2552 (2013).

[12] E. Bagnaschi et al. Eur. Phys. J. C 79, 617 (2019).

[13] H. Bahl, S. Liebler, and T. Stefaniak, Eur. Phys. J. C 79, 279 (2019).

[14] G. Aad et al. (ATLAS and CMS Collaborations), Phys. Rev. Lett. 114, 191803 (2015).

[15] CMS Collaboration, J. High Energy Phys. 11 (2017) 047.

[16] ATLAS Collaboration, Phys. Lett. B 784, 345 (2018).

[17] P. H. Chankowski, S. Pokorski, and J. Rosiek, Nucl. Phys. B423, 437 (1994).

[18] A. Dabelstein, Nucl. Phys. B456, 25 (1995).

[19] D. M. Pierce, J. A. Bagger, K. T. Matchev, and R. J. Zhang, Nucl. Phys. B491, 3 (1997).

[20] M. Frank, T. Hahn, S. Heinemeyer, W. Hollik, H. Rzehak, and G. Weiglein, J. High Energy Phys. 02 (2007) 047.

[21] S. Heinemeyer, W. Hollik, and G. Weiglein, Eur. Phys. J. C 9, 343 (1999).

[22] S. Heinemeyer, W. Hollik, H. Rzehak, and G. Weiglein, Eur. Phys. J. C 39, 465 (2005).

[23] S. Heinemeyer, W. Hollik, H. Rzehak, and G. Weiglein, Phys. Lett. B 652, 300 (2007).

[24] M. Carena, M. Quiros, and C. Wagner, Nucl. Phys. B461, 407 (1996).

[25] M. Carena, H. Haber, S. Heinemeyer, W. Hollik, C. Wagner, and G. Weiglein, Nucl. Phys. B580, 29 (2000).

[26] S. P. Martin, Phys. Rev. D 71, 016012 (2005).

[27] S. Borowka, T. Hahn, S. Heinemeyer, G. Heinrich, and W. Hollik, Eur. Phys. J. C 74, 2994 (2014).
[28] G. Degrassi, S. Di Vita, and P. Slavich, Eur. Phys. J. C 75, 61 (2015).

[29] S. Borowka, S. Paßehr, and G. Weiglein, Eur. Phys. J. C 78, 576 (2018).

[30] R. V. Harlander, P. Kant, L. Mihaila, and M. Steinhauser, Phys. Rev. Lett. 100, 191602 (2008); 101, 039901(E) (2008).

[31] R. Harlander, P. Kant, L. Mihaila, and M. Steinhauser, J. High Energy Phys. 08 (2010) 104.

[32] J. L. Feng, P. Kant, S. Profumo, and D. Sanford, Phys. Rev. Lett. 111, 131802 (2013).

[33] R. V. Harlander, J. Klappert, and A. Voigt, Eur. Phys. J. C 77, 814 (2017).

[34] A. R. Fazio and E. A. Reyes R., Nucl. Phys. B942, 164 (2019).

[35] E. Bagnaschi, G. F. Giudice, P. Slavich, and A. Strumia, J. High Energy Phys. 09 (2014) 092.

[36] P. Draper, G. Lee, and C. E. M. Wagner, Phys. Rev. D 89, 055023 (2014).

[37] G. Lee and C. E. M. Wagner, Phys. Rev. D 92, 075032 (2015).

[38] J. Pardo Vega and G. Villadoro, J. High Energy Phys. 07 (2015) 159.

[39] E. Bagnaschi, J. Pardo Vega, and P. Slavich, Eur. Phys. J. C 77, 334 (2017).

[40] R. V. Harlander, J. Klappert, A. D. Ochoa, and A. Voigt, Eur. Phys. J. C 78, 874 (2018).

[41] E. Bagnaschi, G. Degrassi, S. Paßehr, and P. Slavich, Eur. Phys. J. C 79, 910 (2019).

[42] B. C. Allanach and A. Voigt, Eur. Phys. J. C 78, 573 (2018).

[43] H. Bahl and W. Hollik, J. High Energy Phys. 07 (2018) 182.

[44] B. Allanach, Comput. Phys. Commun. 143, 305 (2002).

[45] A. Djouadi, J.-L. Kneur, and G. Moultaka, Comput. Phys. Commun. 176, 426 (2007).

[46] J. S. Lee, M. Carena, J. Ellis, A. Pilaftsis, and C. E. M. Wagner, Comput. Phys. Commun. 180, 312 (2009).

[47] G. Lee and C. Wagner, MhEFT package, http://gabrlee.com/ code (2016).

[48] P. Athron, J. Park, D. Stöckinger, and A. Voigt, Comput. Phys. Commun. 190, 139 (2015).

[49] P. Athron, J. Park, T. Steudtner, D. Stöckinger, and A. Voigt, J. High Energy Phys. 01 (2017) 079.

[50] P. Athron, M. Bach, D. Harries, T. Kwasnitza, J. Park, D. Stöckinger, A. Voigt, and J. Ziebell, Comput. Phys. Commun. 230, 145 (2018).

[51] F. Staub, Comput. Phys. Commun. 181, 1077 (2010).

[52] F. Staub, Comput. Phys. Commun. 182, 808 (2011).

[53] F. Staub, Comput. Phys. Commun. 184, 1792 (2013).

[54] F. Staub, Comput. Phys. Commun. 185, 1773 (2014).

[55] W. Porod and F. Staub, Comput. Phys. Commun. 183, 2458 (2012). 
[56] F. Staub and W. Porod, Eur. Phys. J. C 77, 338 (2017).

[57] S. Heinemeyer, W. Hollik, and G. Weiglein, Comput. Phys. Commun. 124, 76 (2000).

[58] T. Hahn, S. Heinemeyer, W. Hollik, H. Rzehak, and G. Weiglein, Comput. Phys. Commun. 180, 1426 (2009).

[59] H. Bahl, S. Heinemeyer, W. Hollik, and G. Weiglein, Eur. Phys. J. C 78, 57 (2018).

[60] R. V. Harlander, J. Klappert, and A. Voigt, arXiv:1910.03595.

[61] C. Studerus, Comput. Phys. Commun. 181, 1293 (2010).

[62] D. J. Broadhurst, Z. Phys. C 54, 599 (1992).

[63] J. Fleischer and M. Y. Kalmykov, Phys. Lett. B 470, 168 (1999).

[64] D. J. Broadhurst, Eur. Phys. J. C 8, 311 (1999).

[65] A. I. Davydychev and M. Y. Kalmykov, Nucl. Phys. B699, 3 (2004).

[66] M. Y. Kalmykov, Nucl. Phys. B718, 276 (2005).

[67] M. Y. Kalmykov, J. High Energy Phys. 04 (2006) 056.

[68] V. V. Bytev, M. Y. Kalmykov, and B. A. Kniehl, Nucl. Phys. B836, 129 (2010).

[69] S. P. Martin and D. G. Robertson, Phys. Rev. D 95, 016008 (2017).
[70] A. Freitas, J. High Energy Phys. 11 (2016) 145.

[71] S. Bauberger and A. Freitas, arXiv:1702.02996.

[72] S. Borowka, G. Heinrich, S. P. Jones, M. Kerner, J. Schlenk, and T. Zirke, Comput. Phys. Commun. 196, 470 (2015).

[73] P. Draper and H. Rzehak, Phys. Rep. 619, 1 (2016).

[74] H. Bahl, T. Hahn, S. Heinemeyer, W. Hollik, S. Paßehr, H. Rzehak, and G. Weiglein, arXiv:1811.09073.

[75] T. Hahn, S. Heinemeyer, W. Hollik, H. Rzehak, and G. Weiglein, Phys. Rev. Lett. 112, 141801 (2014).

[76] H. Bahl and W. Hollik, Eur. Phys. J. C 76, 499 (2016).

[77] P. Marquard, L. Mihaila, J. H. Piclum, and M. Steinhauser, Nucl. Phys. B773, 1 (2007).

[78] K. G. Chetyrkin, J. H. Kuehn, and M. Steinhauser, Comput. Phys. Commun. 133, 43 (2000).

[79] B. Schmidt and M. Steinhauser, Comput. Phys. Commun. 183, 1845 (2012).

[80] F. Herren and M. Steinhauser, Comput. Phys. Commun. 224, 333 (2018).

[81] S. P. Martin, Phys. Rev. D 65, 116003 (2002).

[82] I. Jack, D. R. Timothy Jones, S. P. Martin, M. T. Vaughn, and Y. Yamada, Phys. Rev. D 50, R5481 (1994). 